www.jmscr.igmpublication.org

Index Copernicus Value: 79.54

ISSN (e)-2347-176x ISSN (p) 2455-0450

crossref DOI: https://dx.doi.org/10.18535/jmscr/v7i4.55

\title{
Socio-demographic and clinical profile of Parkinsonism patients attending a tertiary care hospital of north India
}

\author{
Authors \\ Dr Shubana Ashraf ${ }^{1^{*}}$, Dr Atif Rasool Kawoosa ${ }^{2}$, Dr Sheikh Mohamad Tahir ${ }^{3}$ \\ ${ }^{1}$ Consultant Neurologist, Super Specialty Hospital, Karanagar, Srinagar \\ ${ }^{2}$ Registrar Neurology, Super Specialty Hospital, Karanagar, Srinagar \\ ${ }^{3}$ Consultant Cardiology, Super Specialty Hospital, Karanagar, Srinagar \\ *Corresponding Author \\ Dr Shubana Ashraf \\ Email: shabana.ashraf79@gmail.com
}

\begin{abstract}
Background: Parkinsonism is a clinical syndrome of akinesia, with or without rigidity, tremor, and postural and gait disturbance. It may be caused by primary degenerative conditions of the brain involving the basal ganglia. We conducted this study with the aim to study the demographic and clinical features of patients with degenerative Parkinsonism presenting to Indraprastha Apollo Hospitals.

Methods: A prospective observational study was conducted at Indraprastha Apollo Hospital between September 2014 and June 2016.A total of 80 patients were enrolled for the study after taking an informed written consent. Among the 80 patients, 40 patients were typical Parkinson's disease patients and the other 40 were atypical Parkinsonism patients.

Results: In this study we recruited a total of 80 patients which included 40 patients of parkinsonism and 40 patients of atypical parkinsonism. Among the parkinsonism patients, 27 (67.5\%) were males, 3 (7.5\%) had family history of parkinsonism and $28(70 \%)$ were on levodopa-carbidopa therapy. The mean age among typical parkinsonism patients was $66.25 \pm 10.55$ (mean $\pm S D$ ), mean age of disease onset was $60.64 \pm 11.76$ and mean duration of the disease was 5.65 \pm 4.54 . The mean Hoehn and Yahr was 2.54.The mean UPDRS (stage 13) was $45.98 \pm 21.98$ and the mean UPDRS (stage 3) was 31.53 \pm 17.39 . According to UPDRS part 1- item 4, the mean apathy score was $0.11 \pm 0.21$ ), the mean HAMD score in typical Parkinsonism was 6.13 \pm 7.66 , and mean MMSE score in was $27.75 \pm 4.64$.

Conclusion: Our study demonstrated socio-demographic and clinical presentation of Parkinsonism patients in our setup. Most common comorbid conditions were also identifies.

Keywords: Parkinsonism, atypical parkinsonism, neurological diseases.
\end{abstract}

\section{Introduction}

Parkinsonism is a clinical syndrome of akinesia, with or without rigidity, tremor, and postural and gait disturbance. It may be caused by primary degenerative conditions of the brain involving the basal ganglia ${ }^{(1)}$. The commonest of these is typical
Parkinson disease (PD) which is predominantly associated with the degeneration of dopaminergic neurons in the substantia nigra. Atypical Parkinsonism refers to a group of conditions that are associated with more widespread neurodegeneration. As a group they present with 
Parkinsonism but are often characterized by early speech and gait impairment, absence of rest tremor, no asymmetry, poor or no response to levodopa and an aggressive clinical course ${ }^{(2)}$. These atypical forms include progressive supranuclear palsy (PSP), dementia with Lewy bodies (DLB), multisystem atrophy (MSA), cortico-basal degeneration (CBD) and others ${ }^{(3)}$. In addition, Parkinsonism may occur secondary to certain drugs or toxins, cerebrovascular disease, encephalitis, anoxic brain injury, demyelination and other lesions affecting the basal ganglia.

In addition to the motor symptoms of bradykinesia, rigidity, tremor, and postural and gait instability, PD patients also show cognitive and psychiatric dysfunction including dementia, mild cognitive impairment (MCI), depression and hallucinations $^{(4)}$. It has now been well observed that the occurrence of symptoms vary among individuals with PD, such that a subgroup of patients may show some symptoms, while another subgroup may show other symptoms. In addition, the complex clinical picture of motor and nonmotor symptoms may change in the same individual as the disease progresses. Interestingly, variability has been seen among PD patients in the rate of progression and the response (good or adverse) to treatment with dopaminergic and other drugs. Classifications of PD into subtypes have been proposed to reduce the heterogeneity of clinical features of PD so as to get a better insight into the neural correlates of this disease and provide better treatment options. Different approaches have been used to identify subtypes of PD based on the age of disease onset, motor and non-motor symptoms and rate of progression ${ }^{(5)}$. Subtype variations may be genetically determined, and therefore may vary in different ethnic groups. To the best of our knowledge, this has not been studied in Indian patients and this forms the basis of this study. We conducted this study with the aim to study the demographic and clinical features of patients with degenerative Parkinsonism presenting to Indraprastha Apollo Hospitals.

\section{Material and Methods}

A prospective observational study was conducted at Indraprastha Apollo Hospital between September 2014 and June 2016.A total of 80 patients were enrolled for the study after taking an informed written consent. Among the 80 patients, 40 patients were typical Parkinson's disease patients and the other 40 were atypical Parkinsonism patients.

Sample size: Sample size was calculated using the Open Epi software for an Group study defined by type of Parkinson Disease keeping an assumption of confidence level at $95 \%$ (i.e. alpha error at 5\%), Power of the study at $80 \%$,odds ratio of 3 that gave a sample size for both group (Atypical and Typical) of 80 by using Flesis method.

Inclusion Criteria: The United Kingdom brain bank criteria (Gibb and Lees 1988) (15) was used to diagnose patients with PD. Appropriate diagnostic criteria was also used to diagnose patients with atypical Parkinsonism.

Exclusion Criteria: Patients with secondary forms of Parkinsonism were excluded such as patients with history of exposure to certain drugs (neuroleptics etc.) or toxins (carbon monoxide etc.); tumor, infection, infarction or osmotic demyelination involving the basal ganglia; hypoxic brain injury; recurrent head injury; hydrocephalus or liver failure.

Procedure: A detailed history was taken including the demographic details, family history and other co-morbid conditions. Disease onset was defined as the time of first recalled motor symptom. Age at onset, disease duration, onset of each motor and non-motor symptom and presence or absence of asymmetry was recorded. A note was made of all the anti-parkinsonian medication being taken, time to initiation of L-dopa treatment and the L-dopa dose.

Motor Symptom Evaluation: Physical examination including neurological examination was performed. Unified Parkinson Disease Rating Scale (UPDRS) ${ }^{(6)}$ was applied to all patients of PD: UPDRS I to IV was done in the off stage and 
UPDRS III was repeated in the on stage. A note was made of response to L-dopa (difference in UPDRS III score in the off and on stage); presence of motor fluctuations and L-dopa induced dyskinesia's and the time to onset of each of these. Disease severity in PD patients was assessed as per the total UPDRS score in the off stage. Rate of total disease progression was calculated as total UPDRS score divided by disease duration. The bradykinesia score was derived by calculating the mean of item numbers 23, 24, 25, 26, 31. The PIGD score was derived by calculating the mean of item number 27, 28, 29, 30 . The tremor score was derived by the mean of item number 20, 21. The total tremor score was derived by dividing the sum of item numbers 16 and 20-21 on the UPDRS scale by 8 . The nontremor score was derived by dividing the sum of the item numbers $5,7,12-15,18,19$ and 22-31 on the UPDRS scale by 26 . The motor phenotype was assessed by the ratio of the tremor to nontremor score (Lewis et al 2003) ${ }^{(7)}$. The subjects were thus divided into three sub types on the basis of their motor phenotypes. The Postural Instability Gait Disorder (PIGD)/ NTD subtype was defined as those subjects whose ratio of total tremor to total non-tremor score was less than or equal to 1 , whereas subjects with a ratio of more than 1.5 was defined as tremor dominant. Subjects with a ratio between 1.0-1.5 were classified as intermediate. The ADL score was calculated by the mean of item number 5 to 17 . The Apathy score was calculated from item number 4 of part 1 of UPDRS. The final assessment of motor phenotype was done after an expert review.

\section{Non- Motor Symptoms}

Mini Mental State Examination (MMSE) (8) and Hamilton's depression rating scale (HAMD) (9) was applied to all PD patients. MMSE score of less than 24 was taken as evidence of dementia. Depressive symptoms were categorized as none if score was less than 8, possible if score 8-20, definite if score was 21-35 and severe if score was more than 35 .

Primary Outcome: The primary outcome was to classify the PD patients into various subtypes based on variables like age of onset, motor or nonmotor phenotype, rate of progression and response to L-dopa using cluster analysis

Secondary Outcome: To divide subjects into three subtypes on the basis of their motor phenotypes, which included the Non-tremor dominant, Tremor dominant and the Intermediate subtypes.

Statistical analysis: The data was entered into Microsoft excel and was analysed using SPSS v.20.0. K One-way analysis of variance (ANOVA), cross-tabulation analysis, and Kruskal-Wallis test were conducted to compare the general characteristics of the subjects. Pairwise comparisons were made using chi-square test for categorical variables and the Wilcoxon rank sum test for continuous variables. Kappa statistical analyses were performed to evaluate the classification consistency between subtype systems. All reported test results were two-tailed and a $P$ value $<0.05$ was considered significant.

Ethical Issues: The study had no ethical issues related to animal or human experimentation.

\section{Results}

Table 1: Socio-demographic characteristics among Parkinsonism and atypical Parkinsonism patients

\begin{tabular}{|l|c|c|c|c|}
\hline \multirow{2}{*}{ Variables } & \multicolumn{2}{c|}{ Parkinsonism } & Atypical Parkinsonism \\
\cline { 2 - 5 } & n & \% & n & \% \\
\hline \multicolumn{4}{|c|}{ Gender } \\
\hline Female & 13 & 32.5 & 15 & 37.5 \\
Male & 27 & 67.5 & 25 & 62.5 \\
Total & $\mathbf{4 0}$ & $\mathbf{1 0 0}$ & $\mathbf{4 0}$ & $\mathbf{1 0 0}$ \\
\hline Family History & 37 & 92.5 & 38 & 95 \\
\hline No & 3 & 7.5 & 2 & 5 \\
Yes & $\mathbf{4 0}$ & $\mathbf{1 0 0}$ & $\mathbf{4 0}$ & $\mathbf{1 0 0}$ \\
\hline
\end{tabular}




\begin{tabular}{|l|c|c|c|c|}
\hline \multicolumn{6}{|l|}{ On Levodopa-carbidopa compound } \\
\hline No & 12 & 30 & 22 & 55 \\
Yes & 28 & 70 & 18 & 45 \\
Total & $\mathbf{4 0}$ & $\mathbf{1 0 0}$ & $\mathbf{4 0}$ & $\mathbf{1 0 0}$ \\
\hline Other Medical History \\
\hline Diabetes mellitus & 3 & 6 & 4 & 10 \\
Hypertension & 7 & 14 & 6 & 15 \\
Hypothyroidism & 2 & 4 & 0 & 0 \\
No & 20 & 60 & 27 & 67 \\
Others & 8 & 16 & 3 & 7.5 \\
Total & 40 & $\mathbf{1 0 0}$ & $\mathbf{4 0}$ & $\mathbf{1 0 0}$ \\
\hline Tremors & \multicolumn{5}{|l}{} \\
\hline Non Tremor & 26 & 52 & & \\
Intermediate Tremor & 7 & 14 & 0 & 0 \\
Tremor & 7 & 14 & & \\
Total & 40 & 100 & & \\
\hline
\end{tabular}

In this study we recruited a total of 80 patients which included 40 patients of typical parkinsonism and 40 patients of atypical parkinsonism. Socio-demographic characteristics of the study patients is shown in table 1.Among the typical parkinsonism patients, 27 (67.5\%) were males, $3(7.5 \%)$ had family history of parkinsonism and 28(70\%) were on levodopacarbidopa therapy. Moreover, associated co- morbid conditions in the typical parkinsonism patients included hypertension and diabetes mellitus in $14 \%$ and $6 \%$ patients respectively. Among the atypical parkinsonism patients, majority patients were males $25(62.5 \%)$, only $2(5 \%)$ had family history of parkinsonism, $18(45 \%)$ were on levodopa-carbidopa therapy, $6(15 \%)$ were hypertensive and only 4(10\%) were having associated diabetes mellitus.

Table 2: Characteristics among typical Parkinsonism patients

\begin{tabular}{|l|c|c|c|c|c|}
\hline Variable & $\mathbf{n}$ & Mean & Standard Deviation & Minimum & Maximum \\
\hline Age & 40 & 66.25 & 10.55 & 48.00 & 90.00 \\
\hline Age Of Onset & 40 & 60.64 & 11.76 & 36.00 & 89.50 \\
\hline Disease Duration Years & 40 & 5.65 & 4.54 & 0.50 & 25.00 \\
\hline Bradykinesia Score & 40 & 1.46 & 0.92 & 0.00 & 4.00 \\
\hline Tremor Score & 40 & 0.61 & 0.54 & 0.00 & 2.28 \\
\hline PIGD Score & 40 & 1.31 & 0.98 & 0.00 & 4.00 \\
\hline Total Tremor Score & 40 & 0.67 & 0.53 & 0.00 & 1.80 \\
\hline Total Non-tremor Score & 40 & 1.18 & 0.72 & 0.14 & 3.20 \\
\hline Motor Phenotype & 40 & 1.06 & 2.03 & 0.00 & 12.40 \\
\hline Severity Score & 40 & 45.98 & 21.98 & 10.00 & 96.00 \\
\hline Disease Progression & 40 & 11.90 & 8.73 & 3.60 & 43.00 \\
\hline ADl Score & 40 & 1.17 & 0.68 & 0.38 & 3.07 \\
\hline UPDRS 3 Off & 40 & 31.53 & 17.39 & 3.00 & 80.00 \\
\hline UPDRS 3 On & 40 & 20.38 & 13.43 & 0.00 & 68.00 \\
\hline Hoehn And Yahr & 40 & 2.54 & 1.13 & 1.00 & 5.00 \\
\hline Global Cognitive Function & 40 & 27.75 & 4.64 & 11.00 & 30.00 \\
\hline HAMD & 40 & 6.13 & 7.66 & 0.00 & 39.00 \\
\hline Apathy & 40 & 0.11 & 0.21 & 0.00 & 0.75 \\
\hline T & & $66.25+10.55$ & & \\
\hline
\end{tabular}

Table 2 shows important characteristics among typical parkinsonism patients. The mean age $66.25 \pm 10.55$ (mean \pm SD), mean age of disease among typical parkinsonism patients was onset was $60.64 \pm 11.76$ and mean duration of the disease was $5.65 \pm 4.54$. The mean Hoehn and 
Yahr was 2.54. The mean disease severity score (was 45.98 \pm 21.98 and the mean UPDRS (stage 3) was $31.53 \pm 17.39$. According to UPDRS part 1item 4 , the mean apathy score was $0.11 \pm 0.21$ ), the mean HAMD score in typical Parkinsonism was $6.13 \pm 7.66$, and mean MMSE score in was 27.75 \pm 4.64 . The mean motor phenotype was $1.06(2.03)$.

Table 3: Characteristics among atypical parkinsonism patients

\begin{tabular}{|l|c|c|c|c|c|}
\hline Variable & $\mathbf{N}$ & Mean & Standard Deviation & Minimum & Maximum \\
\hline Age & 40 & 67.38 & 9.41 & 49 & 85 \\
\hline Age Of Onset & 40 & 64.68 & 8.56 & 44 & 79 \\
\hline Disease Duration Years & 40 & 3.00 & 1.93 & 1 & 8 \\
\hline Severity Score & 40 & 64.28 & 22.94 & 19 & 123 \\
\hline Disease Progression & 40 & 29.63 & 17.37 & 7 & 74 \\
\hline Global Cognitive Function & 40 & 23.58 & 6.90 & 4 & 30 \\
\hline HAMD & 40 & 7.28 & 6.94 & 0 & 27 \\
\hline
\end{tabular}

Table 3 shows important characteristics among atypical parkinsonism patients. The mean age of Atypical Parkinsonism patients was 67.38 \pm 9.41 years, mean age of onset was $64.68 \pm 8.56$ years and the mean duration of the disease was $3 \pm 1.93$ years. The mean UPDRS score of atypical parkinsonism patients (stage 1-3) i.e the severity score was $64.28 \pm 22.94$, the mean HAMD score in atypical patients was $7.28 \pm 6.94$ and the mean MMSE in atypical patients was $23.58 \pm 6.90$.

\section{Discussion}

A prospective observational study was conducted at Indraprastha Apollo Hospital between September 2014 and June 2016. A total of 80 patients were enrolled for the study after taking an informed written consent. The aim of our study was to study the demographic and clinical features of patients with degenerative Parkinsonism presenting to Indraprastha Apollo Hospitals. Among the 80 patients, 40 patients were typical Parkinson's disease patients and the other 40 were atypical Parkinsonism patients.

Among the typical parkinsonism patients, 27 $(67.5 \%)$ were males, $3(7.5 \%)$ had family history of parkinsonism and 28(70\%) were on levodopacarbidopa therapy. Moreover, associated comorbid conditions in the parkinsonism patients included hypertension and diabetes mellitus in $14 \%$ and $6 \%$ patients respectively. Among the atypical parkinsonism patients, 22 patients had a diagnosis consistent with progressive supranuclear palsy, 12 had a diagnosis consistent with multi system atrophy and rest of the 6 patients could not be categorized in any definite category. A majority of patients were males $25(62.5 \%)$, only $2(5 \%)$ had family history of parkinsonism, $18(45 \%)$ were on levodopa-carbidopa therapy and had a poor response to levodopa, 6(15\%) were hypertensive and only 4(10\%) were having associated diabetes mellitus.

The mean motor phenotype was 1.06 indicating that majority of the patients in our study group were of intermediate variety. Based on the motor phenotype, the other subtypes that emerged from our study were Tremor dominant type and nonTremor dominant type. The non-tremor dominant type showed the highest disease severity, mean bradykinesia, mean PIGD score, progression rate and highest cognitive impairment. The tremor dominant types showed the highest tremor to nontremor dominant ratio with a relatively low progression rate that was in accordance with the previous studies ${ }^{(10)}$. In Lewis et al.(2005) study ${ }^{(10)}$ patients were classified as young onset, tremor dominant and non-tremor dominant subtypes. There were about 7 patients in typical parkinsonism patients who presented at an earlier age (mean age of 50.7 year). I another Chinese study ${ }^{(11)}$ patients were alsocategorized into different subtypes.

Non motor symptoms preceded motor symptoms in Parkinson s disease ${ }^{(12)}$. We noted that HAMD score and apathy score, sleep disturbance was 
maximum in non-Tremor dominant subtype. Sensory complaints, fatigue, hallucination were universally present in all subtypes.

The mean age among typical Parkinsonism patients was $66.25 \pm 10.55($ mean \pm SD), mean age of disease onset was $60.64 \pm 11.76$ and mean duration of the disease was $5.65 \pm 4.54$. However, the studies by Gozdek, Laskowska, Michalak, and Gorzelańczyk $^{(13)}$ (2007; $\mathrm{n}=40$, with mean age of 69.7 years), Yamanishi et al. ${ }^{(14)}$ (2013; $\mathrm{n}=117$, with mean age of 69.4 years), and Žiropađa et al. $^{(15)}$ (2009; $\mathrm{n}=102$, with mean age of 58.4 years) are in accordance with the results of our study.

\section{Conclusion}

Our study demonstrated socio-demographic and clinical presentation of Parkinsonism patients in our setup. We also identified clinical heterogeneity in our Indian Parkinson s disease patients.

\section{Source of Funding: None \\ Conflict of Interest: None Declared}

\section{References}

1. DeMaagd G, Philip A. Parkinson's Disease and Its Management: Part 1: Disease Entity, Risk Factors, Pathophysiology, Clinical Presentation, and Diagnosis. $P T$. 2015;40(8):504-532.

2. Williams DR, Litvan I. Parkinsonian syndromes. Continuum (MinneapMinn). 2013;19(5 Movement Disorders):11891212. doi:10.1212/01.CON.000043

3. Wenning GK, Krismer F, Poewe W. New insights into atypical parkinsonism. Curr Opin Neurol. 2011;24(4):331-338. doi:10.1097/WCO.0b013e3283480569 6152.24038.e0

4. Aarsland D, Taylor JP, Weintraub D. Psychiatric issues in cognitive impairment. Mov Disord. 2014;29(5):651662. doi:10.1002/mds. 25873
5. Mostafa AA, Poletti M. Neural and behavioral substrates of subtypes of Parkinson's disease. Frontiers in Systems Neuroscience. 2013;7:1-11.

6. Fahn S, Elton RL. Members of the UPDRS Development Committee. Unified Parkinson's Disease Rating Scale. In: Fahn S, Marsden CD, Calne DB, et al., editors. Recent Developments in Parkinson's Disease. Vol. 2. Florham Park, New Jersey: McMillan Health Care Information; 1987 PP 153-163

7. Lewis SJ, Foltynie T, Blackwell AD, Robbins TW, Owen AM, Barker RA. Heterogeneity of Parkinson's disease in the early clinical stages using a data driven approach. J Neurol Neurosurg Psychiatry. 2005;76(3):343-348. doi: 10.1136/jnnp.2003.033530.

8. Folstein MF, Folstein SE, McHugh PR. "Mini-mental state". A practical method for grading the cognitive state of patients for the clinician. J Psychiatr Res. 1975;12 (3):189-198. doi: 10.1016/00223956(75)90026-6.

9. The Hamilton rating scale for depression, journal of operational psychiatry, 1979;10(2): 149-165.

10. Lewis SJ, Foltynie T, Blackwell AD, Robbins TW, Owen AM, Barker RA. Heterogeneity of Parkinson's disease in the early clinical stages using a data driven approach. J Neurol Neurosurg Psychiatry 2005;76(3):343-348. doi: 10.1136/jnnp.2003.033530.

11. Pinglui , Tao Feng, Bio chen et al; Clinical heterogeneity in patients with early stage Parkinson's disease: a cluster analysis.;J Zhejiang Uni Sci B Sep 2011; v. 12(9) 694-703

12. Chaudhuri, K.R., Naidu, Y., 2008. Early Parkinson's disease and non-motor issues. J. Neurol., 255(s5):33-38. [doi: 10.1007/s00415-008-5006-1] 
13. Gozdek, L., Laskowska, I., Michalak, M., \& Gorzelańczyk, E. (2007). Ocenajakościżyciaosób z chorobą Parkinsona .Polskie Forum Psychologiczne 12(1), 51-62.

14. Yamanishi, T., Tachibana, H., Oguru, M., Matsui, K., Toda, K.,Okuda, B., \& Oka, N. (2013). Anxiety and depression in patients with Parkinson's disease. Internal Medicine 52(5), 539-545.

15. Žiropađa, L., Stefanova, E., Potrebić, A.,\& Kostić,V.S.(2009). Quality of life in Serbian patients with Parkinson's disease. Quality of Life Research,18(7), 833-839. 